\title{
Optica
}

\section{Tight real-time synchronization of a microwave clock to an optical clock across a turbulent air path: supplementary material}

\author{
Hugo Bergeron, ${ }^{1,2 *}$ Laura C. Sinclair, ${ }^{1}$ William C. SWAnn, ${ }^{1}$ Craig W. \\ Nelson, ${ }^{1}$ Jean-Daniel Deschênes, ${ }^{1,2}$ Esther Baumann, ${ }^{1}$ Fabrizio R. \\ Giorgetta, ${ }^{1}$ IAN COddington, ${ }^{1}$ And NATHAN R. NEWBURY ${ }^{1 *}$ \\ ${ }^{1}$ National Institute of Standards and Technology, 325 Broadway, Boulder, Colorado 80305, USA \\ ${ }^{2}$ Université Laval, 2325 Rue de I'Université, Québec, QC, G1V 0A6, Canada \\ *Corresponding authors: hugo.bergeron@nist.gov, nathan.newbury@nist.gov
}

Published 15 April 2016

\begin{abstract}
This document provides supplementary information to "Tight real-time synchronization of a microwave clock to an optical clock across a turbulent air path," http://dx.doi.org/10.1364/optica.3.000441. Here, we discuss details of the microwave clock and present a more detailed schematic.
\end{abstract}

http://dx.doi.org/10.1364/optica.3.000441.s001

It is important to maintain tight phase coherence between the microwave oscillator and the local frequency comb that comprises the microwave clock. The setup is illustrated schematically in Fig. S1. The microwave oscillator is a combination of a quartz oscillator and a dielectric resonator oscillator (DRO). A quiet 100-MHz quartz oscillator is frequency multiplied by a factor of 100 . The DRO is phaselocked to this $10-\mathrm{GHz}$ signal with about $100-\mathrm{kHz}$ bandwidth to reduce the phase noise level at high Fourier frequencies and for greater frequency flexibility. A self-referenced frequency comb is then phaselocked to this signal by mixing the $10.037-\mathrm{GHz}$ output of the Quartz/DRO pair with the detected 50th harmonic of the comb's repetition frequency in a low-noise mixer to create a baseband phase error signal. The digital loop filter (implemented on a fieldprogrammable gate array (FPGA)) that controls these actuators uses a 16-bit ADC to measure the phase error signal and its gains are adjusted to reach a $10-\mathrm{kHz}$ lock bandwidth. Piezoelectric actuators close the feedback loop by changing the repetition frequency of the comb by adjusting the cavity length of the comb. With the feedback loop closed, there are three coherent outputs, one optical and two rf: the optical $200-\mathrm{MHz}$ pulse train, the $10.037-\mathrm{GHz}$ signal generated from direct photodetection of the frequency comb pulses, and the $10.037-\mathrm{GHz}$ signal from the DRO.

With the system described above, the frequency comb is tightly locked to the microwave oscillator. To achieve synchronization, the system then adjusts the phase of the microwave oscillator as described next. First, the time offset is computed from the O-TWTFT master synchronization equation [1] by an FPGA-based controller co-located with the microwave clock. This time offset acts as an in-loop error signal that is filtered (as discussed in Section 3 ) and then used to adjust the frequency of the microwave oscillator. The frequency adjustment is implemented through an offset lock of the DRO to the 10-GHz quartz signal, as shown in Fig. S1. The offset is provided by an adjustable low- noise direct digital synthesizer (DDS) module. A $37 \mathrm{MHz}$ nominal DDS frequency was used, leading to a $10.037-\mathrm{GHz}$ DRO output.

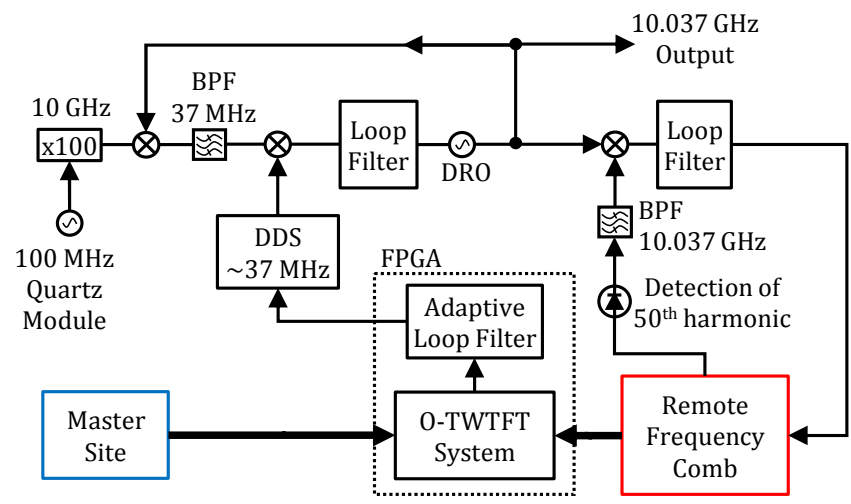

Fig. S1 Stabilization architecture for the microwave clock. BPF: bandpass filter, DRO: dielectric resonator oscillator, O-TWTFT: Optical two-way time-frequency transfer.

\section{REFERENCES}

1. J.-D. Deschenes, L. C. Sinclair, F. R. Giorgetta, W. C. Swann, E. Baumann, H. Bergeron, M. Cermak, I. Coddington, and N. R. Newbury, ArXiv150907888 Phys. (2015). 